\title{
SUFFICIENT CONDITIONS FOR THE INVERSION FORMULA FOR THE $k$-PLANE RADON TRANSFORM IN R ${ }^{n}$
}

\author{
SINE R. JENSEN
}

\begin{abstract}
The inversion theorem (1) for the $k$-plane Radon transform in $\mathrm{R}^{n}$ is often stated for Schwartz functions, cf. [5, p. 110], and lately for smooth functions on $\mathbf{R}^{n}$ fulfilling that $f(x)=O\left(|x|^{-N}\right)$ for some $N>n$, cf. [6, Thm. I.6.2]. In this paper it will be shown, that it suffices to require that $f$ is locally Hölder continuous and $f(x)=O\left(|x|^{-N}\right)$ for some $N>k$ (N not necessarily an integer) in order for (1) to hold, and that the same decay on $f$ but $f$ only continuous implies an inversion formula only slightly weaker than (1).
\end{abstract}

\section{Introduction}

An important area in the theory of the k-plane Radon transform on $\mathrm{R}^{n}$ is the inversion theorems, which gives explicit formulas by which one can recover a function from its $k$-plane transform. Here we shall consider the formula

$$
f=(4 \pi)^{-\frac{k}{2}} \frac{\Gamma\left(\frac{n-k}{2}\right)}{\Gamma\left(\frac{n}{2}\right)} I^{-k}(\hat{f})^{\smile},
$$

where $\hat{f}$ (resp. $\check{f}$ ) denotes the $k$-plane transform (resp. the dual transform) of $f$, while $I^{-k}$ is a Riesz potential, cf. Section 4. It will be shown in this paper, that the formula holds for all functions in the space $C(k, n)$ (see Definition 1.3), and that the formula with $I^{k}$ replaced by $\lim _{\alpha \rightarrow-k_{+}} I^{\alpha}$ holds if $f \in C_{a}\left(\mathrm{R}^{n}\right)$ for some $a>k$.

Notice, that the decay requirement of $C(k, n)\left(f(x)=O\left(|x|^{-N}\right)\right.$ for some $N>k$ ) on its member functions is, in some sense, the weakest possible in order for an inversion formula to hold: A sufficient condition for the integral in the $k$-plane transform of a continuous function $f$ to be convergent is, that for every $k$-plane there exists an $\varepsilon>0$ such that $f(x)=O\left(|x|^{-k-\varepsilon}\right)$ on this $k$-plane. However this non-uniform decrease of $f$ is not enough to make the inversion formula valid. In [14], Zalcman shows the existence of a smooth 
function $f \neq 0$ on $\mathrm{R}^{2}$ satisfying $f(x)=O\left(|x|^{-2}\right)$ on every line, which nonetheless has $\hat{f}=0$. For further examples see e.g. [1] and [2].

The proof in this paper of the inversion formula is rooted in the basic definition of the Riesz potential, $I^{\alpha}(\alpha \in \mathrm{C})$, which is

$$
\left(I^{\alpha} f\right)(x)=\frac{1}{H_{n}(\alpha)} \int_{\mathrm{R}^{n}} f(y)|x-y|^{\alpha-n} d y .
$$

Here $H_{n}$ is a certain meromorphic function. If $f$ is continuous and $O\left(|x|^{-a}\right)$ for some $a>0$, the integral converges if $0<\operatorname{Re} \alpha<a$. For values of $\alpha$ with $\operatorname{Re} \alpha \leq 0$, the Riesz potential can, depending on the regularity of $f$, be defined by analytic continuation (see e.g. [9, sec. 10.2, 10.7] for various ways of performing this extension). The key to the proof of the inversion formula is the identity $I^{-k}\left(I^{k} f\right)=f$, which will be established exactly for $f$ in $C(k, n)$.

Inversion formulas for the Radon transform of $L^{p}$-functions also exists, but then the interpretation of the Riesz potentials is quite different. Examples can be found e.g. in [11] where Rubin verifies two inversion formulas for the case $k=n-1$. One of them is of the same nature as (1), and the other is of the type, where a suitably interpreted Riesz potential in applied before the dual transform instead of after. The last mentioned variant of inversion formula is in [10] proved for $L^{p}$-functions in the case of a general $k$ under the assumption that $1 \leq p<\frac{n}{k}$. It is interesting to note, that given $f \in C\left(\mathrm{R}^{n}\right)$ such that it is $O\left(|x|^{-N}\right)$, then $f \in L^{p}\left(\mathrm{R}^{n}\right)$ when $-N p<-n$, i.e. $p>\frac{n}{N}$. Thus Rubin's inversion formula can be used on this $f$ when there exists a $p \geq 1$ with $\frac{n}{N}<p<\frac{n}{k}$, i.e. when $k<N$ which is precisely the decay condition in the inversion theorem of this paper.

The paper follows the lines of Helgason's exposition [6, Chap. V §5]: After the preliminaries, we study in Section 2 the analytic continuation of the map $\alpha \mapsto x_{+}^{\alpha}(f)=\frac{1}{\Gamma(\alpha+1)} \int_{0}^{\infty} f(x) x^{\alpha} d x$. In Section 3 we use this to study the maps $\alpha \mapsto r^{\alpha}(f)=\frac{1}{\Gamma(\alpha+1)} \int_{\mathrm{R}^{n}} f(x)|x|^{\alpha} d x$, and in Section 4 we introduce Riesz potentials and establish the identity $I^{-k}\left(I^{k} f\right)=f$. Finally, in Section 5 , we prove the two versions of the inversion formula.

The inversion formula in (1), expressed as it is in terms of Riesz potentials, holds for $k$ both odd and even. If $k$ is even it is well-known, that a similar inversion formula can be established using the Laplacian instead of Riesz potentials (see e.g. [6, p. 29]. Section 6 contains a brief discussion of the possible impact of the main result of the paper on the domain of this formula. 


\section{Preliminaries}

For each $a>0$ and $n \in \mathrm{N}$ we make the following definitions:

Definition 1.1. Define the function space $C_{a}\left(\mathrm{R}^{n}\right)$ by

$$
C_{a}\left(\mathrm{R}^{n}\right)=\left\{f \in C\left(\mathrm{R}^{n}\right) \mid f(x)=O\left(|x|^{-a}\right)\right\} .
$$

Definition 1.2. For each $l \in \mathbf{N}_{0}=\mathbf{N} \cup\{0\}, 0<\varepsilon<1$ and $x \in \mathbf{R}^{n}$ define the space $C^{l+\langle\varepsilon\rangle, x}\left(\mathrm{R}^{n}\right)$ as the set of functions $f$ on $\mathbf{R}^{n}$ such that $f$ is $C^{l}$ in some neighborhood $\mathscr{O}$ of $x$ with each $l$ 'th order derivative of $f$ Hölder continuous of index $\varepsilon$ in that neighborhood, i.e.

(2) $\exists M>0 \forall x_{1}, x_{2} \in \mathscr{O} \forall \mathbf{l} \in \mathbf{N}_{0}^{n},|\mathbf{I}|=l:$

$$
\left|\left(\partial^{\mathbf{l}} f\right)\left(x_{1}\right)-\left(\partial^{\mathbf{l}} f\right)\left(x_{2}\right)\right| \leq M\left|x_{1}-x_{2}\right|^{\varepsilon} .
$$

Put

- $C^{l+\langle\varepsilon\rangle}\left(\mathrm{R}^{n}\right)=\bigcap_{x \in \mathrm{R}^{n}} C^{l+\langle\varepsilon\rangle, x}\left(\mathrm{R}^{n}\right) \subset C^{l}\left(\mathrm{R}^{n}\right)$,

- $C^{l+}\left(\mathrm{R}^{n}\right)=\bigcap_{x \in \mathrm{R}^{n}} \bigcup_{\varepsilon>0} C^{l+\langle\varepsilon\rangle, x} \subset C^{l}\left(\mathrm{R}^{n}\right)$

and

- $C_{a}^{l+\langle\varepsilon\rangle, x}\left(\mathrm{R}^{n}\right)=C^{l+\langle\varepsilon\rangle, x}\left(\mathrm{R}^{n}\right) \cap C_{a}\left(\mathrm{R}^{n}\right)$,

- $C_{a}^{l+\langle\varepsilon\rangle}\left(\mathrm{R}^{n}\right)=C^{l+\langle\varepsilon\rangle}\left(\mathrm{R}^{n}\right) \cap C_{a}\left(\mathrm{R}^{n}\right)$

- $C_{a}^{l+}\left(\mathrm{R}^{n}\right)=C^{l+}\left(\mathrm{R}^{n}\right) \cap C_{a}\left(\mathrm{R}^{n}\right)$

- $C_{a}^{l}\left(\mathrm{R}^{n}\right)=C^{l}\left(\mathrm{R}^{n}\right) \cap C_{a}\left(\mathrm{R}^{n}\right)$

Definition 1.3. Finally define for each $k \in\{1, \ldots, n-1\}$ the space $C(k, n)$ as the set of functions $f$, such that $f \in C_{k+\delta}^{0+}\left(\mathrm{R}^{n}\right)$ for some $\delta>0$. I.e. $f \in C(k, n)$ exactly when $f$ is $O\left(|x|^{-k-\delta}\right)$ for some $\delta>0$, and there for each $x \in \mathrm{R}^{n}$ exists a neighborhood $\mathscr{O}$ and an $\varepsilon, 0<\varepsilon<1$, such that $\left|f\left(x_{1}\right)-f\left(x_{2}\right)\right| /\left|x_{1}-x_{2}\right|^{\varepsilon}$ is bounded for $x_{1}, x_{2} \in \mathscr{O}$.

From now on, when the symbols $a, n, l$ and $\varepsilon$ are used, the assumption will be $a>0, n \in \mathrm{N}, l \in \mathrm{N}_{0}$ and $0<\varepsilon<1$, unless otherwise mentioned.

\section{The map $\alpha \mapsto x_{+}^{\alpha}(f)$}

Definition 2.1. For each $\alpha \in \mathrm{C}$ with $-1<\operatorname{Re} \alpha<a-1$ define the map $x_{+}^{\alpha}: C_{a}(\mathrm{R}) \rightarrow \mathrm{C}$ by

$$
x_{+}^{\alpha}(f)=\frac{1}{\Gamma(\alpha+1)} \int_{0}^{\infty} f(x) x^{\alpha} d x .
$$


Remark 2.2. The map $x_{+}^{\alpha}$ is well-defined since $-1<\operatorname{Re} \alpha$ and $f \in$ $C(\mathrm{R})$ makes the integrand integrable at 0 , while $\operatorname{Re} \alpha<a-1$ and $f(x)=$ $O\left(|x|^{-a}\right)$ makes it integrable at $\infty$. Note, that the $\Gamma$-function is a non-vanishing meromorphic function with poles in $-\mathrm{N}_{0}$ and

$$
\lim _{\alpha \rightarrow k}(\alpha-k) \Gamma(\alpha)=\frac{(-1)^{-k}}{(-k) !}, \quad k \in-\mathrm{N}_{0} .
$$

Proposition 2.3. Let $f \in C_{a}^{l+\langle\varepsilon\rangle, 0}(\mathrm{R})$. Then the map $\alpha \mapsto x_{+}^{\alpha}(f)$, defined on

$$
\{\alpha \in \mathrm{C} \mid-1<\operatorname{Re} \alpha<a-1\},
$$

can be (uniquely) extended to a holomorphic map on

$$
\{\alpha \in \mathrm{C} \mid-l-\varepsilon-1<\operatorname{Re} \alpha<a-1\} .
$$

This map will likewise be denoted $\alpha \mapsto x_{+}^{\alpha}(f)$. We have

$$
x_{+}^{\alpha}(f)=(-1)^{(-\alpha-1)} f^{(-\alpha-1)}(0), \quad \text { when } \alpha \in\{-l-1, \ldots,-1\} .
$$

Proof. The integral in (3) is not necessarily convergent in 0 , when $\alpha \leq-1$. But if we put

$$
A(x)=f(x)-\sum_{k=0}^{l} \frac{f^{(k)}(0)}{k !} x^{k} \quad \text { and } \quad B(\alpha)=\sum_{k=0}^{l} \frac{f^{(k)}(0) \rho^{\alpha+k+1}}{k !(\alpha+k+1)} .
$$

then, by calculating the integrals, one realizes that

$$
x_{+}^{\alpha}(f)=\frac{1}{\Gamma(\alpha+1)}\left(\int_{0}^{\rho} x^{\alpha} A(x) d x+\int_{\rho}^{\infty} x^{\alpha} f(x) d x+B(\alpha)\right),
$$

is an extension, cf. [4, p. 57]. Here $0<\rho<1$ fulfills $\overline{B(0, \rho)} \subset \mathcal{O}$, where $\mathcal{O}$ is a neighborhood of 0 in which $f^{(l)}$ is Hölder continuous. This extension is well-defined on

$$
S=\{\alpha \in \mathrm{C} \backslash-\mathrm{N} \mid-l-\varepsilon-1<\operatorname{Re} \alpha<a-1\} .
$$

To show this, only the first term needs thought. Since $f \in C^{l}(\mathcal{O})$, there exists, according to Taylors theorem, for any $x \in B(0, \rho)$ a $y$ between 0 and $x$, such that

$$
f(x)=\sum_{k=0}^{l} \frac{f^{(k)}(0)}{k !} x^{k}+\frac{f^{(l)}(y)-f^{(l)}(0)}{l !} x^{l} .
$$


Because $f^{(l)}$ is Hölder continuous of index $\varepsilon$ in $\mathcal{O}$ we therefore have

$$
\int_{0}^{\rho}\left|x^{\alpha} A(x)\right| d x \leq \text { const } \int_{0}^{\rho} x^{\operatorname{Re} \alpha+l+\varepsilon} d x<\infty
$$

since $\operatorname{Re} \alpha+l+\varepsilon>-l-\varepsilon-1+l+\varepsilon=-1$.

Let $\alpha_{0} \in S$ be given. To show that $\alpha \mapsto x_{+}^{\alpha}(f)$ is holomorphic in $\alpha_{0}$, choose $\delta>0$ such that

$$
B\left(\alpha_{0}, \delta\right) \subset\{\alpha \in \mathrm{C} \backslash-\mathrm{N} \mid-l-\varepsilon-1+\delta<\operatorname{Re} \alpha<a-1-\delta\} .
$$

Clearly $\alpha \mapsto B(\alpha)$ is holomorphic in $\alpha_{0}$. Thus we only need to show, that the two integrals in (6) are holomorphic in $\alpha_{0}$. This will follow from the theorems of Cauchy and Morera, if it can be shown, that for any closed curve $\gamma$ in $B\left(\alpha_{0}, \delta\right)$ the two integrals in each of the following expressions can be interchanged:

$$
\int_{\gamma} \int_{0}^{\rho} x^{\alpha} A(x) \quad \text { and } \quad \int_{\gamma} \int_{\rho}^{\infty} x^{\alpha} f(x) .
$$

But for $x \in] 0, \rho[$

$$
\sup _{\alpha \in B\left(\alpha_{0}, \delta\right)}\left|x^{\alpha} A(x)\right| \leq|A(x)| x^{-l-\varepsilon-1+\delta},
$$

and this function is, as in (8), integrable over ]0, $\rho$ [. For $x \in] \rho, \infty$ [ we have the existence of a constant $c$ independent of $x$, such that

$$
\sup _{\alpha \in B\left(\alpha_{0}, \delta\right)}\left|x^{\alpha} f(x)\right| \leq c x^{a-1-\delta-a}=c x^{-1-\delta} .
$$

Now, let $m \in\{-l-1, \ldots,-1\}$ be given. Choose $\delta^{\prime}>0$ such that

$$
B\left(m, \delta^{\prime}\right) \backslash\{m\} \subset\left\{\alpha \in \mathrm{C} \backslash-\mathrm{N} \mid-l-\varepsilon-1+\delta^{\prime}<\operatorname{Re} \alpha<a-1-\delta^{\prime}\right\} .
$$

As before we have for $\alpha \in B\left(m, \delta^{\prime}\right)$, that

(9) $\left|\int_{0}^{\rho} x^{\alpha} A(x) d x\right| \leq C<\infty \quad$ and $\quad\left|\int_{\rho}^{\infty} x^{\alpha} f(x) d x\right| \leq K<\infty$,

where the constants $C$ and $K$ are independent of $\alpha$. Thus for $\alpha \rightarrow m$ we have

$$
(\alpha-m) \int_{0}^{\rho} x^{\alpha} A(x) d x \rightarrow 0 \quad \text { and } \quad(\alpha-m) \int_{\rho}^{\infty} x^{\alpha} f(x) d x \rightarrow 0 .
$$

Now (5) follows from (6) and (4). 
REMARK 2.4. With the Hölder continuity condition on the derivatives of $f$ replaced by ordinary continuity, the inequality in (8) changes to

$$
\int_{0}^{\rho}\left|x^{\alpha} A(x)\right| d x \leq \text { const } \int_{0}^{\rho} x^{\operatorname{Re} \alpha+l} d x .
$$

Thus when $f \in C_{a}^{l}(\mathrm{R})$, the extension of $\alpha \rightarrow x_{+}^{\alpha}(f)$ still exists but only on

$$
\{\alpha \in C \mid-l-1<\operatorname{Re} \alpha<a-1\} .
$$

\section{The map $\alpha \mapsto r^{\alpha}(f)$}

Definition 3.1. For each $\alpha \in \mathrm{C}$ with $-n<\operatorname{Re} \alpha<a-n$ define the map $r^{\alpha}: C_{a}\left(\mathrm{R}^{n}\right) \rightarrow \mathrm{C}$ by

$$
r^{\alpha}(f)=\frac{1}{\Gamma(\alpha+n)} \int_{\mathbb{R}^{n}}|x|^{\alpha} f(x) d x .
$$

Remark 3.2. As in Remark 2.2 it is seen, that $r^{\alpha}$ is well-defined.

We will express $r^{\alpha}$ by $x_{+}^{\alpha}$. To this end we introduce the mean value function:

Definition 3.3. For any $f \in C\left(\mathrm{R}^{n}\right)$ let $M_{f}: \mathrm{R} \rightarrow \mathrm{C}$ denote the mean value function of $f$ around 0 defined by

$$
M_{f}(t)=\frac{1}{\Omega_{n}} \int_{S^{n-1}} f(t \omega) d \omega
$$

Remark 3.4. Notice, that $t \mapsto M_{f}(t)$ is even, and that $M_{f}(0)=f(0)$.

Lemma 3.5. When $f$ is in $C_{a}^{l+\langle\varepsilon\rangle, 0}\left(\mathrm{R}^{n}\right)$ then $M_{f}$ is in $C_{a}^{l+\langle\varepsilon\rangle, 0}(\mathrm{R})$.

PROOF. Standard arguments.

Remark 3.6. Transition to polar coordinates in the defining expression (10) for $r^{\alpha}$ now gives $r^{\alpha}(f)$ in terms of $x_{+}^{\alpha}$ :

$$
r^{\alpha}(f)=\Omega_{n} x_{+}^{\alpha+n-1}\left(M_{f}\right),
$$

when $-1<\operatorname{Re} \alpha+n-1<a-1$, i.e. $-n<\operatorname{Re} \alpha<a-n$.

Proposition 3.7. Let $f \in C_{a}^{l+\langle\varepsilon\rangle, 0}\left(\mathrm{R}^{n}\right)$. Then the map $\alpha \mapsto r^{\alpha}(f)$, defined on

$$
\{\alpha \in \mathrm{C} \mid-n<\operatorname{Re} \alpha<a-n\},
$$


can be (uniquely) extended to a holomorphic map on

$$
A=\{\alpha \in \mathrm{C} \mid-l-\varepsilon-n<\operatorname{Re} \alpha<a-n\} .
$$

This map will likewise be denoted $\alpha \rightarrow r^{\alpha}(f)$, and it satisfies (12). In specific (13) $r^{\alpha}(f)=\Omega_{n}(-1)^{-\alpha-n} M_{f}^{(-\alpha-n)}(0), \quad$ when $\alpha \in\{-l-n, \ldots,-n\}$.

Proof. Use (12) as definition and apply Proposition 2.3 using Lemma 3.5.

\section{Riesz Potentials}

Definition 4.1. The meromorphic function $H_{n}$ on $\mathrm{C}$ is defined by

$$
H_{n}(\alpha)=2^{\alpha} \pi^{\frac{n}{2}} \frac{\Gamma\left(\frac{\alpha}{2}\right)}{\Gamma\left(\frac{n-\alpha}{2}\right)} .
$$

Remark 4.2. Note that $H_{n}$ has simple poles at each $\alpha \in-2 \mathrm{~N}_{0}$ and a zero in each $\alpha \in n+2 \mathrm{~N}_{0}$.

Definition 4.3. We put $C_{n}=\mathrm{C} \backslash\left(n+2 \mathrm{~N}_{0}\right)$.

Definition 4.4. For each $x \in \mathrm{R}^{n}, f \in C_{a}\left(\mathrm{R}^{n}\right)$, and $\alpha \in \mathrm{C}_{n}$ with $0<$ $\operatorname{Re} \alpha<a$ the $\alpha$ th Riesz potential, $I^{\alpha}$, of $f$ at $x$ is defined as

$$
\begin{aligned}
\left(I^{\alpha} f\right)(x)=\frac{1}{H_{n}(\alpha)} \int_{\mathrm{R}^{n}} f(y) \mid x- & \left.y\right|^{\alpha-n} d y \\
& =\frac{1}{H_{n}(\alpha)} \int_{\mathrm{R}^{n}} f(x-y)|y|^{\alpha-n} d y .
\end{aligned}
$$

Remark 4.5. As in Remark 2.2 it is seen, that $I^{\alpha} f(x)$ is well-defined. Comparing with the defining expression (10) for $r^{\alpha}$ we see, that

$$
\left(I^{\alpha} f\right)(x)=\frac{\Gamma(\alpha)}{H_{n}(\alpha)} r^{\alpha-n}\left(\tau_{x} f\right)
$$

where $\tau_{x} f(y)=f(x-y)$.

Proposition 4.6. Let $x \in \mathbf{R}^{n}$ be given. Assume that $f \in C_{a}^{l+\langle\varepsilon\rangle, x}\left(\mathbf{R}^{n}\right)$. Then the map $\alpha \mapsto\left(I^{\alpha} f\right)(x)$, defined on the set

$$
\left\{\alpha \in \mathrm{C}_{n} \mid 0<\operatorname{Re} \alpha<a\right\},
$$

can be (uniquely) extended to a meromorphic map on

$$
B=\{\alpha \in \mathrm{C} \mid-l-\varepsilon<\operatorname{Re} \alpha<a\} .
$$


This map will likewise be denoted $\alpha \rightarrow\left(I^{\alpha} f\right)(x)$. It satisfies (15) for $\alpha \in$ $B \backslash\left(\left(-\mathrm{N}_{0}\right) \cup\left(n+2 \mathrm{~N}_{0}\right)\right)$. The poles, which are all simple, are in

$$
\left(n+2 \mathrm{~N}_{0}\right) \cup B .
$$

Proof. Use (15) as definition and apply Proposition 3.7 to obtain a (unique) meromorphic extension to $\{\alpha \in \mathrm{C} \mid-l-\varepsilon<\operatorname{Re} \alpha<a\}$. The possible poles are those of $\frac{\Gamma(\alpha)}{H_{n}(\alpha)}=\frac{1}{2} \pi^{-\frac{n+1}{2}} \Gamma\left(\frac{n-\alpha}{2}\right) \Gamma\left(\frac{\alpha+1}{2}\right)$. They are $\alpha \in 2 \mathrm{~N}_{0}+n$ and $\alpha \in-2 \mathrm{~N}_{0}-1$, all simple. When $\alpha \in\left(-2 \mathrm{~N}_{0}-1\right) \cap B$ it follows from (13), that

$$
r^{\alpha-n}(f)=\Omega_{n}(-1)^{-\alpha} M_{f}^{(-\alpha)}(0)=0,
$$

since $M_{f}$ in an even function. Thus $\alpha$ is a removable singularity.

Lemma 4.7. Let $f \in C_{a}^{0+}$. Then $x \mapsto\left(I^{0} f\right)(x)$ is defined on all of $\mathrm{R}^{n}$ and

$$
I^{0} f=f \text {. }
$$

Proof. It follows from Proposition 4.6, that $x \mapsto\left(I^{0} f\right)(x)$ is defined on all of $\mathbf{R}^{n}$. Since

$$
\lim _{\alpha \rightarrow 0} \alpha H_{n}(\alpha)=\frac{2 \pi^{\frac{n}{2}}}{\Gamma\left(\frac{n}{2}\right)}=\Omega_{n},
$$

it follows from Proposition 4.6, (13), (4) and Remark 3.4, that

$$
\left(I^{0} f\right)(x)=\lim _{\alpha \rightarrow 0} \frac{\alpha \Gamma(\alpha)}{\alpha H_{n}(\alpha)} r^{\alpha-n}\left(\tau_{x} f\right)=M_{\tau_{x} f}(0)=f(x) .
$$

Lemma 4.8. Let $f \in C_{a}\left(\mathrm{R}^{n}\right)$. Let $\alpha \in \mathrm{C}$ with $0<\operatorname{Re} \alpha<\min (a, n)$ be given. Then

$$
I^{\alpha} f \in C_{b-\operatorname{Re} \alpha}\left(\mathrm{R}^{n}\right),
$$

for any $b$ with $\operatorname{Re} \alpha<b \leq \min (a, n)$ if $a \neq n$, andfor any $b$ with $\operatorname{Re} \alpha<b<n$ if $a=n$.

Proof. See [6, Prop. V.5.8.] with natural modifications to the proof in case $a=n$.

Proposition 4.9. Let $f \in C_{a}\left(\mathrm{R}^{n}\right)$. For any pair $\alpha, \beta \in \mathrm{C}$ satisfying

$\operatorname{Re} \alpha>0$ and $\operatorname{Re} \beta>0$ and $\operatorname{Re}(\alpha+\beta)<\min (a, n)$ we have

$$
I^{\alpha} I^{\beta} f=I^{\alpha+\beta} f .
$$


Remark 4.10. Refer to e.g. [7, p. 43ff] or [8, Satz 9] in order to see how, when dealing with Riesz potentials as distributions, (17) can be expressed as a convolution of distributions. The distribution approach can prove Proposition 4.9 for a smaller class of functions.

Proof. That $0<\operatorname{Re} \beta<\min (a, n)$ implies two things. First we get from Remark 4.5, that $I^{\beta} f$ is well-defined and given by

$$
\left(I^{\beta} f\right)(z)=\frac{1}{H_{n}(\beta)} \int_{\mathrm{R}^{n}} f(y)|z-y|^{\beta-n} d y .
$$

Secondly, we get the usage of Lemma 4.8 from which follows, that

$$
I^{\beta} f \in C_{b-\operatorname{Re} \beta}\left(\mathbf{R}^{n}\right),
$$

where $b$ is chosen such that $\operatorname{Re}(\alpha+\beta)<b<\min (a, n)$. Thus, because $0<\operatorname{Re} \alpha<b-\operatorname{Re} \beta, I^{\alpha}\left(I^{\beta} f\right)$ is well-defined and given by

$$
\begin{aligned}
I^{\alpha}\left(I^{\beta} f\right)(x) & =\frac{1}{H_{n}(\alpha)} \int_{\mathrm{R}^{n}}\left(I^{\beta} f\right)(z)|x-z|^{\alpha-n} d z \\
& =\frac{1}{H_{n}(\alpha)} \frac{1}{H_{n}(\beta)} \int_{\mathrm{R}^{n}} \int_{\mathrm{R}^{n}} f(y)|z-y|^{\beta-n} d y|x-z|^{\alpha-n} d z .
\end{aligned}
$$

To show, that the order of integration can be interchanged, consider the expression

$$
\int_{\mathrm{R}^{n}}|f(y)| \int_{\mathrm{R}^{n}}|z-y|^{\operatorname{Re} \beta-n}|x-z|^{\operatorname{Re} \alpha-n} d z d y .
$$

By substituting $v=\frac{x-z}{|x-y|}$ in the inner integral and using the rotation invariance of the Lebesgue measure, this expression is rewritten as

$$
\int_{\mathrm{R}^{n}}|f(y)||x-y|^{\operatorname{Re} \alpha+\operatorname{Re} \beta-n} d y \int_{\mathrm{R}^{n}}|e-v|^{\operatorname{Re} \beta-n}|v|^{\operatorname{Re} \alpha-n} d v,
$$

where $e$ is an arbitrary fixed unit vector. Now $0<\operatorname{Re}(\alpha+\beta)<a$ makes the $y$-integral convergent. That the $v$-integral is convergent can be seen easily. Finally, it can be shown, e.g. using Fourier transform as in [13, p. 117-118], that

$$
\int_{\mathrm{R}^{n}}|e-v|^{\beta-n}|v|^{\alpha-n} d v=\frac{H_{n}(\alpha) H_{n}(\beta)}{H_{n}(\alpha+\beta)} .
$$


REMARK 4.11. Let $x_{0} \in \mathrm{R}^{n}$ be given. In what follows, we will often decompose a given function $f$ on $\mathbf{R}^{n}$ as $f=f_{1}+f_{2}$, where $f_{1}=(1-\chi) f$ and $f_{2}=\chi f$ for some compactly supported $C^{\infty}$-function $\chi$ with $\chi(x)=1$ in some neighborhood of $x_{0}$. Note that $f_{1}$ and $f_{2}$ have the same regularity as $f$, but $f_{1}$ is 0 in the neighborhood of $x_{0}$ and $f_{2}$ has compact support.

Lemma 4.12. Let $f \in C_{a}^{l}\left(\mathrm{R}^{n}\right)$. Let $\alpha \in \mathrm{C}_{n}$ with $0<\operatorname{Re} \alpha<a$ and $x_{0} \in \mathrm{R}^{n}$ be given. Write $f=f_{1}+f_{2}$ as in Remark 4.11. Then $I^{\alpha} f_{1}$ is smooth at $x_{0}$, and $I^{\alpha} f_{2} \in C^{l}\left(\mathbf{R}^{n}\right)$ with $\partial^{\mathbf{p}}\left(I^{\alpha} f_{2}\right)=I^{\alpha}\left(\partial^{\mathbf{p}} f_{2}\right)$ for any $\mathbf{p} \in \mathbf{N}_{0}^{n}$ with $|\mathbf{p}| \leq l$.

Proof. Assume $\mathbf{p} \in \mathrm{N}_{0}^{n}$ to be given. Choose $\delta>0$ such that $f_{1}=1$ in $B\left(x_{0}, 2 \delta\right) \subset \mathcal{U}$. Then for any $x \in B\left(x_{0}, \delta\right)$

$$
\begin{aligned}
\left|f_{1}(y) \partial_{x}^{\mathbf{p}}\right| x-\left.y\right|^{\alpha-n} \mid & \leq c\left|f_{1}(y)\right||x-y|^{\operatorname{Re} \alpha-n-|\mathbf{p}|} \\
& \leq c^{\prime} 1_{\mathrm{R}^{n} \backslash B\left(x_{0}, 2 \delta\right)}(y)(|y|+1)^{-a}\left|y-x_{0}\right|^{\operatorname{Re} \alpha-n-|\mathbf{p}|},
\end{aligned}
$$

since $\frac{1}{2}\left|x_{0}-y\right| \leq|x-y| \leq 2\left|x_{0}-y\right|$ for $y \notin B\left(x_{0}, 2 \delta\right)$. Here $c^{\prime}$ does not depend on $x$. Since $-a+\operatorname{Re} \alpha-n-|\mathbf{p}|<-a+a-n=-n$, this gives us an integrable majorant of $\partial_{x}^{\mathbf{p}}\left(f_{1}(y)|x-y|^{\alpha-n}\right)$ and it is independent of $x$.

To deal with $I^{\alpha} f_{2}$ assume $|\mathbf{p}| \leq l$ and let $\mathcal{N}$ be any bounded subset of $\mathbf{R}^{n}$. Let $x \in \mathscr{N}$. Then

$$
\begin{aligned}
\left|\partial_{x}^{\mathbf{p}}\left(f_{2}(x-y)|y|^{\alpha-n}\right)\right| & =\left|\left(\partial^{\mathbf{p}} f_{2}\right)(x-y)\right||y|^{\operatorname{Re} \alpha-n} \mid \\
& \leq \sup \left|\partial^{\mathbf{p}} f_{2}\right| 1_{\mathcal{N}+(-\mathscr{C})}(y)|y|^{\operatorname{Re} \alpha-n}, \quad \forall y \in \mathbf{R}^{n} .
\end{aligned}
$$

Since $\operatorname{Re} \alpha>0$ this is an integrable majorant of $\partial_{x}^{\mathbf{p}}\left(f_{2}(x-y)|y|^{\alpha-n}\right)$ and it is independent of $x$. Thus $\partial^{\mathbf{p}}\left(I^{\alpha} f_{2}\right)$ exists in $\mathcal{N}, \mathcal{N}$ arbitrary, and thus in all of $\mathbf{R}^{n}$, and we see from (20) that $\partial^{\mathbf{p}}\left(I^{\alpha} f_{2}\right)=I^{\alpha}\left(\partial^{\mathbf{p}} f_{2}\right)$.

Lemma 4.13. Let $f \in C_{a}^{l}\left(\mathrm{R}^{n}\right)$. Let $\alpha \in \mathrm{C}_{n}$ with $0<\operatorname{Re} \alpha<a$ be given. Then

$$
I^{\alpha} f \in C^{l}\left(\mathrm{R}^{n}\right)
$$

and for any $x \in \mathrm{R}^{n}$ and $0<\varepsilon<1$

$$
f \in C^{l+\langle\varepsilon\rangle, x}\left(\mathrm{R}^{n}\right) \Rightarrow I^{\alpha} f \in C^{l+\langle\varepsilon\rangle, x}\left(\mathrm{R}^{n}\right) .
$$

Proof. Let $x_{0} \in \mathbf{R}^{n}$ be given. Write $f=f_{1}+f_{2}$ as in Remark 4.11. From the preceeding lemma $I^{\alpha} f_{1}$ is smooth at $x_{0}$ and $I^{\alpha} f_{2} \in C^{l}\left(\mathrm{R}^{n}\right)$. Thus (21) holds.

Assume now, that $f \in C^{l+\langle\varepsilon\rangle, x_{0}}\left(\mathrm{R}^{n}\right)$. Let $\mathbf{l} \in \mathrm{N}_{0}^{n}$ with $|\mathbf{I}|=l$ be given. To show the Hölder continuity of $\partial^{\mathbf{I}}\left(I^{\alpha} f_{2}\right)\left(=I^{\alpha}\left(\partial^{\mathbf{l}} f_{2}\right)\right.$ according to Lemma 4.12), 
let $\mathscr{K}$ be a compact neighborhood of $x_{0}$ in which $\partial^{\mathbf{l}} f$ is Hölder continuous of index $\varepsilon$ and assume $\chi$ in the decomposition $f=f_{1}+f_{2}=(1-\chi) f+\chi f$ to have $\mathscr{K}$ as its support. Then $\partial^{\mathbf{l}} f_{2}$ is Hölder continuous of index $\varepsilon$ in all of $\mathrm{R}^{n}$, so for any bounded neighborhood $\mathcal{N}$ of $x_{0}$ and any $x_{1}, x_{2} \in \mathcal{N}$

$$
\begin{aligned}
& \left|\partial^{\mathbf{l}}\left(I^{\alpha} f_{2}\right)\left(x_{1}\right)-\partial^{\mathbf{l}}\left(I^{\alpha} f_{2}\right)\left(x_{2}\right)\right| \\
& \leq \frac{1}{H_{n}(\alpha)} \int_{\mathcal{N}+(-\mathscr{H})}\left|\partial^{\mathbf{l}} f_{2}\left(x_{1}-y\right)-\partial^{\mathbf{l}} f_{2}\left(x_{2}-y\right)\right||y|^{\operatorname{Re} \alpha-n} d y \leq M^{\prime}\left|x_{1}-x_{2}\right|^{\varepsilon}
\end{aligned}
$$

for some $M^{\prime}>0$.

Lemma 4.14. Let $f \in C_{a}\left(\mathrm{R}^{n}\right)$. Let $\alpha \in \mathrm{C}_{n}$ with $\operatorname{Re} \alpha=1$ be given. If $a>1$ then

$$
f \in C^{l+\langle\varepsilon\rangle, x}\left(\mathrm{R}^{n}\right) \Rightarrow \forall \varepsilon^{\prime}, 0<\varepsilon^{\prime}<\varepsilon: I^{\alpha} f \in C^{(l+1)+\left\langle\varepsilon^{\prime}\right\rangle, x}\left(\mathrm{R}^{n}\right)
$$

for any $x \in \mathrm{R}^{n}$ and $0<\varepsilon<1$.

Proof. Let $x_{0} \in \mathrm{R}^{n}$ be given. Write $f=f_{1}+f_{2}$ as in Remark 4.11. Then from Lemma $4.12 I^{\alpha} f_{1}$ is smooth at $x_{0}$, so only $I^{\alpha} f_{2}$ needs thought.

Pick $\mathbf{p} \in \mathrm{N}_{0}^{n}$ with $|\mathbf{p}|=l+1$. Write $\mathbf{p}=\mathbf{I}+e_{i}$ for some $\mathbf{l} \in \mathrm{N}_{0}^{n}$ with $|\mathbf{I}|=l$, and some $e_{i}=(0, \ldots, 0,1,0, \ldots, 0)$. Let $\mathscr{K}$ be a compact neighborhood of $x_{0}$ in which $\partial^{l} f$ is Hölder continuous and assume $\chi$ in the decomposition $f=f_{1}+f_{2}=(1-\chi) f+\chi f$ to have $\mathscr{K}$ as its support. Put $g=\partial^{\mathbf{l}} f_{2}$. Then $g$ is Hölder continuous of index $\varepsilon$ in all of $\mathbf{R}^{n}$ and has support in $\mathscr{K}$. What needs to be shown is, that $\partial^{\mathbf{p}}\left(I^{\alpha} f_{2}\right)=\partial_{i} \partial^{\mathbf{I}}\left(I^{\alpha} f_{2}\right)=\partial_{i}\left(I^{\alpha} g\right)$ (Lemma 4.12) exists and is Hölder continuous in a neighborhood of $x_{0}$.

Let $B$ be a symmetric, bounded neighborhood of 0 such that $\mathscr{K} \subset B+x=$ $B_{x}$ for all $x$ in some bounded, open neighborhood $\mathscr{O}$ of $x_{0}$. Let $\beta \in \mathrm{C}$ with $1<\operatorname{Re} \beta<2$ be given. Then for any $x \in \mathscr{O}$

$$
\begin{aligned}
\partial_{i} I^{\beta} g(x) & =c_{n}(\beta) \int_{B_{x}} g(y)\left(x_{i}-y_{i}\right)|x-y|^{\beta-n-2} d y \\
& =c_{n}(\beta) \int_{B} g(x-y) y_{i}|y|^{\beta-n-2} d y
\end{aligned}
$$

where $c_{n}(\beta)=\frac{\beta-n}{H_{n}(\beta)}$ and where the integral exists since $\operatorname{Re} \beta>1$ and $B$ is bounded. Furthermore, using the Hölder continuity of $g$

$$
\left.\left.\int_{B}\left|(g(x-y)-g(x)) y_{i}\right| y\right|^{\alpha-n-2}\left|d y \leq M \int_{B}\right| y\right|^{-n+\varepsilon} d y<\infty,
$$


i.e. the integral $\int_{B}(g(x-y)-g(x)) y_{i}|y|^{\alpha-n-2} d y$ exists. Using the symmetry of $B$ we get

$$
\begin{aligned}
& \text { (24) }\left.\left|\frac{1}{c_{n}(\beta)} \partial_{i} I^{\beta} g(x)-\int_{B}(g(x-y)-g(x)) y_{i}\right| y\right|^{\alpha-n-2} d y \mid \\
& \leq \int_{B}\left|(g(x-y)-g(x)) y_{i}\left(|y|^{\beta-n-2}-|y|^{\alpha-n-2}\right)\right| d y+\left.\left|g(x) \int_{B} y_{i}\right| y\right|^{\beta-n-2} d y \mid \\
& \leq\left. c^{\prime} \int_{B}|| y\right|^{\beta-n-1+\varepsilon}-|y|^{\alpha-n-1+\varepsilon} \mid d y
\end{aligned}
$$

for some $c^{\prime}>0$. Now notice that when $n=1$, then $c_{n}$ has a removable singularity at $\beta=1$, so that for any value of $n \in \mathrm{N}, c_{n}$ is bounded and bounded away from 0 in a small enough neighborhood of $\alpha$, i.e. $\lim _{\beta \rightarrow \alpha} \frac{1}{c_{n}(\beta)}$ exists and is not 0 . Thus (24) shows that in the limit where $\operatorname{Re} \beta>1$

$$
\lim _{\beta \rightarrow \alpha} \partial_{i} I^{\beta} g(x)=c_{n}(\alpha) \int_{B}(g(x-y)-g(x)) y_{i}|y|^{\alpha-n-2} d y
$$

uniformly on $\mathcal{O}$. So $\partial_{i} I^{\alpha} g$ does exist and

$$
\partial_{i} I^{\alpha} g=c_{n}(\alpha) \int_{B}(g(x-y)-g(x)) y_{i}|y|^{\alpha-n-2} d y
$$

in all of $\mathcal{O}$. Given $0<\varepsilon^{\prime}<\varepsilon$ put $s=\frac{\varepsilon^{\prime}}{\varepsilon}$ and $t=1-s$. We then have for any $x_{1}, x_{2} \in \mathcal{O}$, that

$$
\begin{aligned}
& \left|\partial_{i} I^{\alpha} g\left(x_{1}\right)-\partial_{i} I^{\alpha} g\left(x_{2}\right)\right| \\
= & \left.c_{n}(\alpha)\left|\int_{B}\left(g\left(x_{1}-y\right)-g\left(x_{1}\right)-\left(g\left(x_{2}-y\right)-g\left(x_{2}\right)\right)\right) y_{i}\right| y\right|^{\alpha-n-2} d y \\
\leq & c_{n}(\alpha) \int_{B}\left|\left(g\left(x_{1}-y\right)-g\left(x_{1}\right)\right)-\left(g\left(x_{2}-y\right)-g\left(x_{2}\right)\right)\right|^{t} \\
& \quad\left|\left(g\left(x_{1}-y\right)-g\left(x_{2}-y\right)\right)-\left(g\left(x_{1}\right)-g\left(x_{2}\right)\right)\right|^{s}|y|^{-n} d y \\
\leq & c_{n}(\alpha) \int_{B}\left(2 M|y|^{\varepsilon}\right)^{t}\left(2 M\left|x_{1}-x_{2}\right|^{\varepsilon}\right)^{s}|y|^{-n} d y \\
= & c_{n}(\alpha)\left|x_{1}-x_{2}\right|^{\varepsilon s} 2 M \int_{B}|y|^{\varepsilon t-n} d y=M^{\prime}\left|x_{1}-x_{2}\right|^{\varepsilon^{\prime}}
\end{aligned}
$$

for some constant $M^{\prime}>0$.

CoRollary 4.15. Let $f \in C_{a}\left(\mathrm{R}^{n}\right)$. Let $\alpha \in \mathrm{C}$ with $0<\operatorname{Re} \alpha<\min (a, n)$ be given. Then

$$
f \in C^{l+\langle\varepsilon\rangle, x}\left(\mathrm{R}^{n}\right) \Rightarrow \forall \varepsilon^{\prime}, 0<\varepsilon^{\prime}<\varepsilon: I^{\alpha} f \in C^{(l+[\operatorname{Re} \alpha])+\left\langle\varepsilon^{\prime}\right\rangle, x}\left(\mathrm{R}^{n}\right)
$$


for any $x \in \mathrm{R}^{n}$ and $0<\varepsilon<1$. Here $[\operatorname{Re} \alpha]$ denotes the integer part of $\operatorname{Re} \alpha$.

Proof. Write $\alpha=\beta+[\operatorname{Re} \alpha]$. Then $0 \leq \operatorname{Re} \beta<1$. From Proposition 4.9 combined with Lemma 4.8

$$
I^{\alpha} f=I^{\beta}\left(I^{1}\left(I^{1}\left(\ldots\left(I^{1} f\right) \ldots\right)\right),\right.
$$

$I^{1}$ applied $[\operatorname{Re} \alpha]$ times. The claim now follows from Lemma 4.14 and Lemma 4.13 .

Proposition 4.16. Let $k \in\{1, \ldots, n-1\}$ and $f \in C(k, n)$. Then

$$
I^{-k}\left(I^{k} f\right)=f \text {. }
$$

Proof. Let $x \in \mathrm{R}^{n}$ be given and choose $\delta, 0<\delta<1$, such that $f \in$ $C_{k+\delta}\left(\mathrm{R}^{n}\right)$. From Proposition 4.6 it follows, that there exists an $\delta^{\prime}, 0<\delta^{\prime}<1$, such that the map

$$
\alpha \mapsto\left(I^{\alpha+k} f\right)(x)
$$

is holomorphic in $\left\{\alpha \in \mathrm{C} \mid-k-\delta^{\prime}<\operatorname{Re} \alpha<\delta\right\}$. Since Lemma 4.8 and Corollary 4.15 with $a=b=k+\delta$ ensures, that

$$
I^{k} f \in C_{\delta}^{k+}\left(\mathrm{R}^{n}\right),
$$

we likewise get from Proposition 4.6, that there exists a $\delta^{\prime \prime}, 0<\delta^{\prime \prime}<1$, such that the map

$$
\alpha \mapsto\left(I^{\alpha}\left(I^{k} f\right)\right)(x)
$$

is well-defined and holomorphic in $\left\{\alpha \in \mathrm{C} \mid-k-\delta^{\prime \prime}<\operatorname{Re} \alpha<\delta\right\}$. Proposition 4.9 gives us, that

$$
I^{\alpha} I^{k} f(x)=I^{\alpha+k} f(x),
$$

when $\alpha \in\{\alpha \in \mathrm{C} \mid 0<\operatorname{Re} \alpha<\delta\}$. By analytic continuation this identity then holds on all of $\left\{\alpha \in \mathrm{C} \mid-k-\min \left(\delta^{\prime}, \delta^{\prime \prime}\right)<\operatorname{Re} \alpha<\delta\right\}$. In particular, using Lemma 4.7 with $a=k+\delta$

$$
I^{-k} I^{k} f(x)=I^{0} f(x)=f(x) .
$$

\section{The Inversion Formula for the Radon Transform}

Let $k \in\{1, \ldots, n-1\}$ be given. Let $f \in C_{a}\left(\mathrm{R}^{n}\right)$ for some $a>k$. For the $k$-plane transform one arrives, by calculating, at

$$
(\hat{f})^{\swarrow}(x)=(4 \pi)^{\frac{k}{2}} \frac{\Gamma\left(\frac{n}{2}\right)}{\Gamma\left(\frac{n-k}{2}\right)}\left(I^{k} f\right)(x),
$$


cf. [3] or [6, p. 29]. This will be used in what follows.

Theorem 5.1. Let $k \in\{1, \ldots, n-1\}$. Assume, that $f \in C(k, n)$. Then $f$ can be recovered from its $k$-plane transform by

$$
f=(4 \pi)^{-\frac{k}{2}} \frac{\Gamma\left(\frac{n-k}{2}\right)}{\Gamma\left(\frac{n}{2}\right)} I^{-k}(\hat{f})^{\smile} .
$$

Proof. The claim follows from (25) by means of Proposition 4.16.

Remark 5.2. Any differentiable function will also be locally Hölder continuous (but the inverse implication is not true). Thus the Hölder condition could in the entire paper have been replaced by demanding all functions to be one more time continuously differentiable. E.g. Theorem 5.1 is true for all $f \in C^{1}\left(\mathrm{R}^{n}\right)$ with $f(x)=O\left(|x|^{-k-\delta}\right)$ for some $\delta>0$.

An even lower regularity requirement on $f$ can be bought at a small price:

Theorem 5.3. Let $k \in\{1, \ldots, n-1\}$. Assume, that $f \in C_{k+\delta}$ for some $\delta>0$. Then $f$ can be recovered from its $k$-plane transform by

$$
f=(4 \pi)^{-\frac{k}{2}} \frac{\Gamma\left(\frac{n-k}{2}\right)}{\Gamma\left(\frac{n}{2}\right)} \lim _{s \rightarrow-k_{+}} I^{s}(\hat{f})^{\sim} .
$$

We will need the following lemma pointed out to me by Boris Rubin (cf. $[12$, Thm. I.2.6]):

Lemma 5.4. Let $f \in C_{a}(\mathrm{R})$. Then

$$
\lim _{s \rightarrow-1_{+}} x_{+}^{s}(f)=f(0) \text {. }
$$

Proof. Let $\varepsilon>0$ be given and choose $\delta, 0<\delta<1$, such that $\mid f(x)-$ $f(0) \mid \leq \varepsilon$ when $|x| \leq \delta$. Write

$$
=\frac{1}{\Gamma(s+1)}\left[\int_{0}^{\delta}(f(x)-f(0)) x^{s} d x+\int_{\delta}^{\infty} f(x) x^{s} d x+\int_{0}^{\delta} f(0) x^{s} d x\right] .
$$

Since (when $s>-1$ ),

$$
\left|\frac{1}{\Gamma(s+1)} \int_{0}^{\delta}(f(x)-f(0)) x^{s} d x\right| \leq \frac{\varepsilon}{\Gamma(s+2)} \delta^{s+1}
$$


and (when $s-a<-1$ )

$$
\left|\frac{1}{\Gamma(s+1)} \int_{\delta}^{\infty} f(x) x^{s} d x\right| \leq \frac{c}{\Gamma(s+1)|s-a+1|} \delta^{s-a+1}
$$

for some constant $c>0$ and

$$
\left|\frac{1}{\Gamma(s+1)} \int_{0}^{\delta} f(0) x^{s} d x-f(0)\right| \leq\left|f(0)\left(\frac{\delta^{s+1}}{\Gamma(s+2)}-1\right)\right|,
$$

$\left|x_{+}^{s}(f)-f(0)\right|$ can be estimated by e.g. some multiple of $\varepsilon$ when $s$ is sufficiently close to -1 .

Also a parallel of Corollary 4.15 and thus of Lemma 4.14 for functions with the Hölder continuity of the derivatives replaced by ordinary continuity is needed:

Lemma 5.5. Let $f \in C_{a}^{l}\left(\mathrm{R}^{n}\right)$. Let $\alpha \in \mathrm{C}_{n}$ with $\operatorname{Re} \alpha=1$ be given. If $a>1$, then

$$
I^{\alpha} f \in C^{l+\langle\varepsilon\rangle}\left(\mathrm{R}^{n}\right)
$$

for any $0<\varepsilon<1$.

Proof. Let $x \in \mathbf{R}^{n}$ and $0<\varepsilon<1$ be given. Decompose $f=f_{1}+f_{2}$ as in Remark 4.11. Then $I^{\alpha} f_{1}$ is smooth at $x$ according to Lemma 4.12, so only $I^{\alpha} f_{2}$ needs thought.

From Lemma $4.12 I^{\alpha} f_{2}$ is in $C^{l}\left(\mathbf{R}^{n}\right)$ with $\partial^{\mathbf{l}} I^{\alpha} f_{2}=I^{\alpha} \partial^{\mathbf{l}} f_{2}$ for any $\mathbf{l} \in \mathbf{N}_{0}^{n}$ with $|\mathbf{I}|=l$. The claim is, that these derivatives are Hölder continuous of index $\varepsilon$ at $x$. Therfore pick $\mathscr{O}$, a bounded neighborhood of $x$, and $x_{1}, x_{2} \in \mathscr{O}$. Since $f_{2}$ has compact support $\mathscr{K}$, there exists $c>0$ such that

$$
\left|\partial^{\mathbf{l}} I^{\alpha} f_{2}\left(x_{1}\right)-\partial^{\mathbf{l}} I^{\alpha} f_{2}\left(x_{2}\right)\right| \leq c \int_{\mathscr{C}}|| x_{1}-\left.y\right|^{\alpha-n}-\left|x_{2}-y\right|^{\alpha-n} \mid d y .
$$

Thus it suffices to prove the existence of a constant $C>0$ (independent of $x_{1}$ and $x_{2}$ ) such that

$$
\int_{\mathscr{K}}|| x_{1}-\left.y\right|^{\alpha-n}-\left|x_{2}-y\right|^{\alpha-n}|d y \leq C| x_{1}-\left.x_{2}\right|^{\varepsilon} .
$$

Put

$$
B_{1}=B\left(x_{1}, \frac{2}{3}\left|x_{1}-x_{2}\right|\right) \quad B_{2}=B\left(x_{2}, \frac{2}{3}\left|x_{1}-x_{2}\right|\right) \quad A=\mathscr{K} \backslash\left(B_{1} \cup B_{2}\right) .
$$


Then $\mathscr{K} \subset B_{1} \cup B_{2} \cup A$, so (26) holds if it can be proved with $\mathscr{K}$ replaced by each of the three sets $B_{1}, B_{2}$ and $A$. But since $\left|x_{2}-y\right|>\frac{1}{3}\left|x_{1}-x_{2}\right|$ when $y \in B_{1}$

$$
\begin{aligned}
\int_{B_{1}}|| x_{1}-\left.y\right|^{\alpha-n}-\left|x_{2}-y\right|^{\alpha-n} \mid d y \\
\quad \leq \int_{B_{1}}\left|x_{1}-y\right|^{1-n} d y+\int_{B_{1}}\left|x_{2}-y\right|^{1-n} d y \\
\quad \leq \int_{B\left(0, \frac{2}{3}\left|x_{1}-x_{2}\right|\right)}|y|^{1-n} d y+\int_{B\left(0, \frac{2}{3}\left|x_{1}-x_{2}\right|\right)}\left(\frac{1}{3}\left|x_{1}-x_{2}\right|\right)^{1-n} d y \\
\quad \leq C_{1}\left|x_{1}-x_{2}\right| .
\end{aligned}
$$

An equivalent calculation can be done for the integral on $B_{2}$. Thus we turn to the integral on $A$.

First let $y \in A$ with $\left|x_{1}-y\right| \neq\left|x_{2}-y\right|$ be given. Apply the mean value theorem to the function $t \mapsto \operatorname{Re} t^{\alpha-n}$ on the interval with endpoints $\left|x_{1}-y\right|$ and $\left|x_{2}-y\right|$ to obtain the existence of an $\left.s_{1} \in\right] 0,1[$ such that

$$
\begin{aligned}
|\operatorname{Re}| x_{1}-\left.y\right|^{\alpha-n} & -\operatorname{Re}\left|x_{2}-y\right|^{\alpha-n} \mid \\
& \leq c^{\prime}\left(s_{1}\left|x_{1}-y\right|+\left(1-s_{1}\right)\left|x_{2}-y\right|\right)^{-n}|| x_{1}-y|-| x_{2}-y|| .
\end{aligned}
$$

Then apply the mean value theorem to the function $t \mapsto \operatorname{Im} t^{\alpha-n}$ to obtain an $s_{2}$ and a similar evaluation of $|\operatorname{Im}| x_{1}-\left.y\right|^{\alpha-n}-\operatorname{Im}\left|x_{2}-y\right|^{\alpha-n} \mid$. Conclude from this that for any $y \in A$

$$
|| x_{1}-\left.y\right|^{\alpha-n}-\left|x_{2}-y\right|^{\alpha-n}\left|\leq c^{\prime \prime}\left(\min \left(\left|x_{1}-y\right|,\left|x_{2}-y\right|\right)\right)^{-n}\right| x_{1}-x_{2} \mid .
$$

Choose $K>0$ such that $B\left(y_{1}, K\right) \cap B\left(y_{2}, K\right) \supset A$ for all $y_{1}, y_{2} \in \mathscr{O}$. Then $K$ is independent of $x_{1}$ and $x_{2}$ and

$$
\begin{aligned}
& \int_{A}\left(\min \left(\left|x_{1}-y\right|,\left|x_{2}-y\right|\right)\right)^{-n} d y \\
\leq & \int_{B\left(x_{1}, K\right) \backslash B_{1}}\left|x_{1}-y\right|^{-n} d y+\int_{B\left(x_{2}, K\right) \backslash B_{2}}\left|x_{2}-y\right|^{-n} d y \\
\leq & 2 \Omega_{n}\left(\log K-\log \left(\frac{2}{3}\left|x_{1}-x_{2}\right|\right)\right) \leq C^{\prime}\left(1+\left|x_{1}-x_{2}\right|^{\varepsilon-1}\right),
\end{aligned}
$$

where $C^{\prime}$ is independent of $x_{1}$ and $x_{2}$. The last evaluation holds because $\varepsilon-1<$ 0 . 
Corollary 5.6. Let $f \in C_{a}^{l}\left(\mathrm{R}^{n}\right)$. Let $\alpha \in \mathrm{C}$ with $1 \leq \operatorname{Re} \alpha<\min (a, n)$ be given. Then

$$
I^{\alpha} f \in C^{(l+[\operatorname{Re} \alpha]-1)+\langle\varepsilon\rangle}\left(\mathrm{R}^{n}\right)
$$

for any $0<\varepsilon<1$.

Proof. Let $0<\varepsilon<1$ be given. If $\operatorname{Re} \alpha=1$ the claim is the previous lemma. If $\operatorname{Re} \alpha>1$ write $\alpha=\beta+1$. From Proposition 4.9

$$
I^{\alpha} f=I^{\beta}\left(I^{1} f\right) .
$$

According to the previous lemma $I^{1} f \in C^{l+\langle\varepsilon\rangle}\left(\mathrm{R}^{n}\right)$, so the claim follows from Corollary 4.15.

Proof of Theorem 5.3. Use Remark 2.4 to modify the conclusions of Proposition 3.7 and 4.6 regarding the set of definition of the extension when the Hölder continuity on the derivatives of $f$ is replaced by ordinary continuity. Use this in following the lines of the proof of Proposition 4.16: Let $x \in \mathrm{R}^{n}$ be given. The map

$$
\alpha \mapsto\left(I^{\alpha+k} f\right)(x)
$$

is holomorphic in $\{\alpha \in \mathrm{C} \mid-k<\operatorname{Re} \alpha<\delta\}$. Since Lemma 4.8 and Corollary 5.6 ensures, that

$$
I^{k} f \in C_{\delta}^{(k-1)+\langle\varepsilon\rangle}\left(\mathrm{R}^{n}\right)
$$

for all $0<\varepsilon<1$, the map

$$
\alpha \mapsto\left(I^{\alpha}\left(I^{k} f\right)\right)(x)
$$

is holomorphic in

$$
\bigcup_{0<\varepsilon<1}\{\alpha \in \mathrm{C} \mid-(k-1+\varepsilon)<\operatorname{Re} \alpha<\delta\}=\{\alpha \in \mathrm{C} \mid-k<\operatorname{Re} \alpha<\delta\} .
$$

Thus by Proposition 4.9 and analytic extension

$$
I^{\alpha}\left(I^{k} f\right)(x)=I^{\alpha+k} f(x),
$$

when $-k<\operatorname{Re} \alpha<\delta$. The last step of the proof of Proposition 4.16 requires Lemma 4.7 the conclusion of which does not hold for an arbitrary $f \in C_{a}\left(\mathrm{R}^{n}\right)$ $\left(I^{0}(f)\right.$ does not necessarily exist). But we can use Lemma 5.4 to replace Lemma 4.7 by (see (16))

$$
\begin{aligned}
\lim _{s \rightarrow 0_{+}}\left(I^{s} f\right)(x) & =\lim _{s \rightarrow 0_{+}} \frac{\Gamma(s)}{H_{n}(s)} r^{s-n}\left(\tau_{x} f\right) \\
& =\lim _{s \rightarrow-1_{+}} x_{+}^{s}\left(M_{\tau_{x} f}\right)=M_{\tau_{x} f}(0)=f(x) .
\end{aligned}
$$


Thus, using (27), we have that

$$
\lim _{s \rightarrow-k_{+}} I^{s}\left(I^{k} f(x)\right)=f .
$$

This in connection with (25) proves the theorem.

\section{The Inversion Formula in Terms of the Laplacian}

It is known, cf. [6], that if $k$ is even, the inversion formula can be stated by means of the Laplacian, $\Delta$, instead of the more complicated Riesz potentials. In fact

THEOREM 6.1. When $k$ is even, and $f \in C^{2}\left(\mathrm{R}^{n}\right)$, and $f$ and all its first and second order derivatives are $O\left(|x|^{-k-\varepsilon}\right)$ for some $\varepsilon>0$, then

$$
f=(4 \pi)^{-\frac{k}{2}} \frac{\Gamma\left(\frac{n-k}{2}\right)}{\Gamma\left(\frac{n}{2}\right)}(-\Delta)^{\frac{k}{2}}(\hat{f})^{\check{2}} .
$$

Proof. Follow the lines of [6, p. 16-17]: First notice that it suffices for $f$ to be continuous and $O\left(|x|^{-k-\varepsilon}\right.$ ) for some $\varepsilon>0$ in order to have formula [6, (34)] for the $k$-plane transform; that is,

$$
(\hat{f})^{\llcorner}(x)=\Omega_{k} \int_{0}^{\infty} F(r, x) r^{k-1} d r
$$

for any $x \in \mathrm{R}^{n}$, where $F(r, x)=\frac{1}{\Omega_{n}} \int_{S^{n-1}} f(x+r \omega) d \omega$. Here $d \omega$ is the Haar measure on the unit sphere $S^{n-1}$ in $\mathrm{R}^{n}$ with total mass $\Omega_{n}=\frac{2 \pi^{\frac{n}{2}}}{\Gamma\left(\frac{n}{2}\right)}$. Then notice, that the demands on the decay of the derivatives of $f$ allows us to apply the Laplacian (with respect to $x$ ) on (29) by interchanging it with the integration. By means of Darboux's equation, it can now be seen, as in [6, p. 16-17], that

$$
\Delta\left((\hat{f})^{\longleftarrow}\right)(x)=\left\{\begin{array}{ll}
-\Omega_{k}(n-k) f(x) & k=2 \\
-\Omega_{k}(n-k)(k-2) \int_{0}^{\infty} F(r, x) r^{k-3} d r & k \neq 2
\end{array} .\right.
$$

When $k=2$ this is (28). For $k \neq 2$ the expression is similar to (29) - the power of $r$ in the integral has just been reduced and it is still larger than -1. Thus the Laplacian can be applied once more without inducing further demands on $\mathrm{f}$ or its derivatives. Continued iteration proves (28).

Can the Theorem 5.1 be used to enlarge the class of functions for which (28) holds? Not much, I think. Some relevant thoughts are the following: Let $\alpha_{0} \in \mathrm{C}$ be given. Using Definition 4.4 and Green's formula it is not hard to 
see, that for $\varphi \in C^{2}\left(\mathrm{R}^{n}\right)$ with sufficient decay of $\varphi$ and all it's first and second order derivatives $\left(O\left(|x|^{-2-\varepsilon}\right)\right.$ for some $\varepsilon>0$ is enough),

$$
I^{\alpha} \Delta \varphi=-I^{\alpha-2} \varphi
$$

in some strip $\{\alpha \in \mathrm{C} \mid 2<\operatorname{Re} \alpha<2+\delta\}$. If furthermore $\varphi \in C^{l+}\left(\mathrm{R}^{n}\right)$ for some integer $l \geq-\operatorname{Re} \alpha_{0}+2$, Proposition 4.6 can be used to extend both sides of (30) holomorphically to $\alpha_{0}$ and thus prove (30) for $\alpha=\alpha_{0}$.

Iterating (30) and then using Lemma 4.7 proves that when $k$ is even and positive, and $h \in C^{k+}\left(\mathrm{R}^{n}\right)$, and $h$ and all it's derivatives of order less than or equal to $k$ have a certain decay ( $O\left(|x|^{-2-\varepsilon}\right)$ for some $\varepsilon>0$ is enough), then

$$
(-\Delta)^{\frac{k}{2}} h=I^{-k} h \text {. }
$$

Thus we see from Theorem 5.1 that (28) holds also for $f \in C(k, n)$ when, in stead of decay demands on derivatives of $f$, we demand a certain decay of $(\hat{f})^{2}$ and all it's derivatives of order less than or equal to $k\left(O\left(|x|^{-2-\varepsilon}\right)\right.$ is enough). Notice, that since $(\hat{f})^{\vee}$ is proportional to $I^{k} f$ the derivatives of $(\hat{f})^{\vee}$ do exist according to Corollary 4.15.

ACKNOWLEDGMENTS. My sincere thanks to Sigurdur Helgason and Boris Rubin for their highly useful suggestions and comments during my work with this paper. Also thanks to Henrik Schlichtkrull for all the time he spent commenting on the paper.

\section{REFERENCES}

1. Armitage, D. H., A non-constant continuous function on the plane whose integral on every line is zero, Amer. Math. Monthly 101 (1994), 892-894.

2. Armitage, D. H., and Goldstein, M., Nonuniqueness for the Radon transform, Proc. Amer. Math. Soc. 117 (1993), 175-178.

3. Fuglede, Bent, An integral formula, Math. Scand. 6 (1958), 207-212.

4. Gelfand, I. M., and Schilow, G. E., Verallgemeinerte Funktionen, VEB Deutscher Verlag der Wissenschaften, 1960.

5. Helgason, Sigurdur, Groups and Geometric Analysis (reprint), Amer. Math. Soc., 2000.

6. Helgason, Sigurdur, The Radon Transform, 2. edition, Birkhäuser, Boston, Basel, Berlin, 1999.

7. Landkof, N. S., Foundations of Modern Potential Theory, Grundlehren Math. Wiss. 180 (1972).

8. Ortner, Norbert, Faltung hypersingulärer Integraloperatoren, Math. Ann. 248 (1980), 19-46.

9. Rubin, Boris, Fractional Integrals and Potentials, Addison Wesley Longman, Essex, U.K., 1996.

10. Rubin, Boris, Inversion of k-plane transforms via continuous wavelet transforms, J. Math. Anal. Appl. 220 (1998), 187-203.

11. Rubin, Boris, Inversion of Radon transforms using wavelet transforms generated by wavelet measures, Math. Scand. 85 (1999), 285-300. 
12. Samko, S. G., Kilbas, A. A., and Marichev, O. I., Fractional Integrals and Derivatives, Gordon and Breach Sc. Publ., 1993.

13. Stein, Elias M., Singular Integrals and Differentiability Properties of Functions, Princeton Univ. Press, Prinston, NJ, 1970.

14. Zalcman, Lawrence, Uniqueness and nonuniqueness for the Radon transform, Bull. London Math. Soc. 14 (1982), 241-245.

DEPARTMENT OF MATHEMATICS

UNIVERSITY OF COPENHAGEN

UNIVERSITETSPARKEN 5

DK-2100 COPENHAGEN $\varnothing$

DENMARK

E-mail: sine@math.ku.dk 